Culture et histoire dans l'espace roman

9 | 2012

Empreintes / emprunts dans le monde hispanique

\title{
La trace du cinéma franquiste dans La buena nueva d'Helena Taberna : un cas remarquable d'intericonicité
}

Nicolas Blayo

\section{(2) OpenEdition}

Journals

Édition électronique

URL : https://journals.openedition.org/cher/11456

DOI : $10.4000 /$ cher. 11456

ISSN : 2803-5992

Éditeur

Presses universitaires de Strasbourg

Édition imprimée

Date de publication : 1 décembre 2012

Pagination : 91-98

ISBN : 978-2-35410-049-0

ISSN : 1968-035X

Référence électronique

Nicolas Blayo, "La trace du cinéma franquiste dans La buena nueva d'Helena Taberna : un cas

remarquable d'intericonicité », reCHERches [En ligne], 9 | 2012, mis en ligne le 14 février 2022, consulté

le 16 février 2022. URL : http://journals.openedition.org/cher/11456 ; DOI : https://doi.org/10.4000/ cher. 11456 


\title{
La trace du cinéma franquiste dans La buena nueva d'Helena Taberna: un cas remarquable d'intericonicité
}

\author{
NiCOLAS BLAYO \\ Université Paul Verlaine - Metz
}

\begin{abstract}
A l'instar des autres créations artistiques, la genèse d'œuvres cinématographiques est tributaire des productions antérieures qui nourrissent et favorisent l'éclosion de nouvelles formes d'expression. Cela semble être une évidence qui a pourtant parfois du mal à être énoncée clairement, tant cette dépendance pose question et échappe souvent à une partie du public. Quelles en sont les manifestations, quels en sont les effets, quel en est le but, si tant est qu'il y en ait un, tout cela doit nous interroger à l'heure de l'analyse de film. "Tout texte est un intertexte», écrit Roland Barthes, «d'autres textes sont présents en lui, à des niveaux variables, sous des formes plus ou moins reconnaissables: les textes de la culture antérieure et ceux de la culture environnante; tout texte est un tissu nouveau de citations révolues» (Barthes 1999: 1015). Dans le cadre de notre réflexion sur les empreintes et les emprunts, il nous a semblé intéressant de nous pencher sur les aspects que prennent les différentes modalités de l'intertextualité dans un long-métrage espagnol sorti en 2008, La Buena Nueva, de la réalisatrice navarraise Helena Taberna. Dans une certaine mesure, ce film nous paraît constituer une sorte d'épitomé des formes intertextuelles et, s'agissant d'une œuvre iconographique, des relations d'intericonicité, selon la typologie énoncée par Gérard Genette, dans Palimpsestes. La littérature au second degré, qui parle de "relation de coprésence entre deux ou plusieurs textes" (Genette 1982: 9-14). De la pratique de la citation au plagiat en passant
\end{abstract}


par l'allusion et le paratexte, ces catégories de l'intertextualité sont comme méthodiquement déclinées. La réalisatrice présente d'ailleurs son travail comme un hypertexte, c'est-à-dire, toujours selon Gérard Genette «tout texte dérivé d'un texte antérieur par transformation simple (nous dirons désormais transformation tout court) ou par transformation indirecte: nous dirons imitation». Toute la question réside dans l'identification du ou des textes antérieurs.

Dans La Buena Nueva, un jeune prêtre, Miguel, frais émoulu du séminaire, est nommé curé d'un village de Navarre, à la veille du soulèvement militaire de juillet 1936 qui déclenchera la guerre civile. L'évêque, père spirituel et ami, lui a confié la mission difficile de ramener sur le droit chemin les brebis égarées, c'est-à-dire la majorité des habitants, ouvertement socialistes et anticléricaux. Il va gagner leur confiance en s'opposant, au nom du message évangélique, à la violence de la répression exercée par la Phalange et les Carlistes qui se sont emparés du village dès le premier jour du soulèvement. Mais il sera désavoué par son évêque et incompris par ses anciens camarades qui, désormais, s'entraînent militairement au séminaire pour participer à ce que les militaires séditieux appelaient «la Croisade». Acculé, il abandonnera le village en compagnie d'une jeune veuve et de sa fille.

La Buena Nueva entre dans le genre du film d'époque ou film historique et, à cet égard, il s'inscrit particulièrement bien dans cette articulation entre différentes œuvres, car sa mise en scène intègre les représentations antérieures de l'histoire qui conditionnent et dessinent en partie l'horizon d'attente du spectateur, constitué dans la définition donnée par Hans Robert Jauss: «[...] d'attentes, de souvenirs, d'anticipations et qui détermine et délimite la signification» de l'œuvre (Jauss 1978: 78).

\section{Le paratexte et l'hypertexte}

Le premier contact du spectateur avec le film est toujours la découverte du titre qui peut déjà faire jouer une modalité intertextuelle, soit la relation entretenue entre l'œuvre et son paratexte, ici le titre, «la bonne nouvelle»,

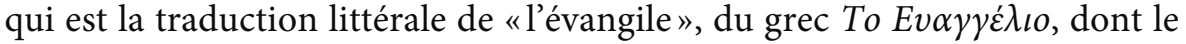
sens moral et la signification eschatologique se détacheront clairement dans ce film, davantage que la dimension historique ou spirituelle. La conception éthique de l'exercice de son ministère empêchera Miguel de cesser d'assister les victimes. Le titre fonctionne à la fois comme une énigme et comme l'anticipation de l'action de Miguel. Le film s'ouvre sur une séquence en 
montage alterné où l'on voit, d'une part, Miguel et l'évêque jouer à la pelote basque dans le cloître du séminaire et, d'autre part, des images en gros plan de cierges qui évoquent des explosifs placés dans des caisses. La dichotomie de l'innocence et de la violence tapie dans l'institution elle-même se dessine déjà. La courte séquence s'achève sur un dérapage et une chute proleptique de Miguel qui n'a pas vu venir une feinte de son adversaire gaucher. L'évêque l'aide fraternellement à se relever pour lui annoncer une bonne nouvelle: sa nomination dans le village fictif d'Alzania.

Helena Taberna présente le film comme étant librement inspiré des mémoires de son oncle, Marino Ayerra, qui fut nommé curé d'Alsasua le 16 juillet 1936. Son texte, intitulé No me avergoncé del Evangelio, publié à Buenos Aires en 1958, relate une histoire très semblable à celle du personnage de Miguel. La transformation, l'une des modalités de l'hypertextualité, est mise en œuvre de manière consciente et, pourtant, le nom de Marino Ayerra n'est pas mentionné dans les génériques de début et de fin. Le spectateur est simplement informé que le film est «una historia inspirada en hechos reales». Le terme est faible pour un scénario qui reprend l'essentiel du récit de l'oncle et presque à la lettre des phrases-clef du livre comme celle-ci, que prononce l'évêque en annonçant sa nomination dans ce village de gauche: «Usted allí, más de izquierdas que nadie, ya me entiende» (Ayerra 1958:23). Phrase qui devient dans le film: «que si son de izquierdas, el nuevo párroco es el más rojo de todos, ¿me entiende? ». Mais les emprunts ne se limitent pas à ce seul témoignage, fondamental, et sont bien plus cinématographiques que livresques, bien davantage artistiques qu'historiques ce qui, tout bien pesé, est parfaitement cohérent avec la nature même de l'œuvre, qui est une fiction et non un documentaire, qui est audio-visuelle et non textuelle.

\section{De la citation cinéphilique à l'allusion cinématographique}

L'arrivée du nouveau curé à Alzania, dès la sixième minute, suscite la gentille provocation de trois hommes assis qui entonnent, au son d'un accordéon, les paroles de la version satirique et anticléricale de l'Hymne de Riego, l'hymne officiel de la République: «Si los curas y frailes supieran/ la paliza que les van a dar, / subirían al coro cantando: "Libertad, libertad, libertad!" " Derrière eux, trône une affiche du Cine popular qui annonce La Mère (Mamb), de Poudovkine. Cette citation de ce film de 1926, bien connu des cinéphiles dès les années 1930 et qui fut projeté en Espagne à cette époque, participe certes de l'effet de réel et de l'ancrage historique. Mais elle fonctionne bien davantage comme indice de l'évolution du 
cheminement intellectuel de Miguel qui, comme le personnage de la Mère chez Poudovkine, verra qu'il ne peut avoir confiance en la parole de l'autorité des nouveaux hiérarques du village. Le destin individuel, approche privilégiée par le cinéaste russe, est aussi la démarche adoptée par Helena Taberna. Le choix de La Mère comme citation indicielle nous semble d'ailleurs doublement pertinente dans la mesure où la thématique du film soviétique - la promesse de clémence trahie - rejoint le film de Francesc Betriu, Réquiem por un campesino español (1985), adaptation du roman de Ramón J. Sender (1950), film auquel il est clairement fait allusion dans plusieurs scènes, en particulier celle de l'affrontement verbal entre le curé et le capitaine phalangiste et celle de l'exécution nocturne des Républicains capturés en présence du curé effondré.

Helena Taberna a déclaré (Huete Machado 2008) avoir pensé à faire $L a$ Buena Nueva en voyant aussi le film Amen de Costa-Gavras (2002) dans lequel un jésuite tente vainement de faire réagir le Vatican face à la Shoah. Mais, en dehors du fait que la référence soit quelque peu disproportionnée, il nous semble qu'elle ne pourrait s'appliquer qu'à la séquence du repas au cours duquel Miguel constate le refus de l'évêque de s'opposer aux exécutions sommaires. En revanche, les emprunts à un film franquiste, $L a$ guerra de Dios (Hommes en détresse), nous paraissent bien plus pertinents à analyser. Ce film, sorti en 1953, écrit par Vicente Escrivá et réalisé par Rafael Gil, cinéaste du régime tombé dans un relatif oubli pour cette raison même, est exemplaire du cinéma social clérical dont Balarrasa (José Antonio Nieves Conde, 1951), également écrit par Escrivá, est le plus connu. Le cinéma clérical, écrit Jean-Claude Seguin, "offre l'image d'une Espagne fer de lance du catholicisme» (Seguin 1994: 45). Film empreint d'une foi indéfectible en l'avenir, La guerra de Dios est à opposer à Surcos (José Antonio Nieves Conde, 1951), film du néo-réalisme ibérique chargé de pessimisme, qui a fortement influencé la production cinématographique espagnole ultérieure.

La guerra de Dios raconte l'histoire d'un brillant séminariste qui est nommé, à sa grande surprise, curé d'un petit village de mineurs où les haines entre la classe ouvrière et les patrons suscitent le désarroi de leurs enfants. Le curé est sommé de choisir son camp par le cacique local mais il refuse de rejeter les mineurs qu'il va assister dans leur lutte pour la justice. Un drame dans la mine, où deux enfants sont accidentellement emmurés, crée les conditions de la réconciliation sous l'égide du curé. Le film présente un scénario de lutte des classes, ce qui peut surprendre dans le contexte $\mathrm{du}$ franquisme, mais la religion va permettre de surmonter les divisions et réunir les âmes. Refusé par la censure dans un premier temps, le scénario 
placera l'action dans les années 1930 - ce qui en fait un film d'époque - pour éviter les complications et le film bénéficiera du label "película de interés nacional», ce qui lui assurera une bonne carrière commerciale mais sera aussi très bien reçu par la critique, y compris à l'étranger. On voit déjà, dans les grandes lignes, de sérieuses similitudes avec le film d'Helena Taberna. Notons au passage que La guerra de Dios est lui-même un film très riche en liens intertextuels et intericoniques, que nous ne développerons pas ici, puisqu'il est très fortement influencé par deux films américains, Qu'elle était verte ma vallée (How Green Was My Valley) de John Ford (1941) et La Citadelle (The Citadel) de King Vidor (1938) et aussi par Journal d'un curé de campagne de Robert Bresson (1951) auquel Rafael Gil va jusqu'à emprunter l'acteur belge Claude Laydu, désormais spécialisé dans les rôles de curés. Par effet de contiguïté, on trouvera des ressemblances entre le film de Bresson, lui-même adapté du roman de Georges Bernanos (1936), et La buena nueva, sans passer par La guerra de Dios qui évacue certains éléments, l'écriture $\mathrm{du}$ journal intime en particulier mais aussi les rapports ambigus entre le curé et la jeune femme. Pour Michael Riffaterre, l'intertextualité qui est le «phénomène qui oriente la lecture du texte, qui en gouverne éventuellement l'interprétation, et qui est le contraire de la lecture linéaire» (Riffaterre 1981: 5) est essentielle pour appréhender la question de la réception de l'œuvre qui est vue de façon d'autant plus subjective qu'elle est reliée à d'autres productions antérieures. D'autres éléments sont sur une frontière mal définie entre citation et allusion, par exemple le rôle du football dans le processus de séduction des enfants pour surmonter l'hostilité des adultes, dans un but réconciliateur chez Gil et Taberna, ou inscrite dans l'expression de la perversion du prêtre chez Almodóvar dans La mala educación (2004). L'intericonicité joue pleinement et interroge le spectateur sur le sens qu'il donne aux images ce qui, en définitive, est l'intérêt ultime de ces emprunts.

\section{Un grand emprunt très catholique}

La trace de Rafael Gil dépasse la simple allusion dans le film d'Helena Taberna qui puise dans La guerra de Dios tellement d'éléments scénaristiques qu'on pourrait être tenté de parler de plagiat de certaines scènes, c'est-à-dire une reprise littérale, même si le concept lui-même est parfois difficile à cerner à tel point que Jean Giraudoux, en 1928, affirme que "le plagiat est la base de toutes les littératures, excepté de la première qui d'ailleurs est inconnue» (Giraudoux 1949: 6). Borges, lui aussi, énonce dans Ficciones (1944): «No 
existe el concepto del plagio: se ha establecido que todas las obras son obra de un solo autor, que es intemporal y es anónimo" (Borges 1997: 12).

Les emprunts très catholiques de La buena nueva à La guerra de Dios, ainsi qu'aux autres œuvres citées, sont nombreux et, néanmoins, le film s'articule de façon autonome, comme œuvre nouvelle et originale. Dans les deux films, l'action s'ouvre sur le séminaire où le spectateur assiste à la nomination du jeune curé qui, et c'est là l'essentiel, place le message évangélique au-dessus de tout le reste. C'est particulièrement souligné dans La guerra de Dios où l'on entend la fin du discours sur la justice sociale que prononce le futur curé du village minier. Cette foi inébranlable en l'Évangile ne peut que conduire à l'accomplissement du Bien dans le film franquiste. En revanche, le jeune curé idéaliste chez Helena Taberna se heurte fatalement à l'engagement fasciste de l'Église espagnole en 1936, jusqu'à en tirer les conséquences; il devra abandonner la prêtrise, contraint et forcé. La trace du film de Rafael Gil nous accompagne tout au long de La Buena Nueva où l'on peut établir un parallèle entre les mineurs anticléricaux, d'une part, et les veuves des Républicains assassinés, d'autre part, deux groupes qui accepteront in fine la médiation du curé, tant son engagement en leur faveur est sincère. On est loin de l'incompréhension qui, dans le film de Bresson, habite le curé d'Ambricourt face à l'hostilité des paroissiens et qui s'interroge: «que leur ai-je donc fait?». Dans nos deux films, le curé espagnol protège les victimes de la répression et se veut un Juste, au sens où l'entend Paul Ricœur, en tant

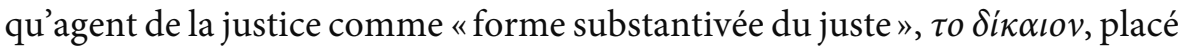
au sommet des vertus et pour qui «l'idée du juste n'est autre que l'idée du bon considéré dans le rapport à autrui » (Ricœur 2004: 217-218). Si ce n'est pas très surprenant sous la plume de Vicente Escrivá, cela peut le sembler davantage dans le film de Taberna. Mais ce serait oublier la spécificité d'une partie du clergé d'Euskal Herria qui a également souffert de la répression franquiste (Sánchez-Vallejo 2007). Miguel dresse clandestinement une cartographie des fosses communes des environs du village dans un but mémoriel, d'une «juste mémoire» chère à Ricœur et, lors de son départ, fait de son enfant de chœur le dépositaire du secret qu'il devra transmettre aux générations futures. Nous sommes là en pleine prise avec l'actualité du débat autour de la mémoire historique, débat qui suscite une grande production de publications et de films; la sortie, deux mois avant celle de La Buena Nueva, de Los Girasoles ciegos, de José Luis Cuerda, est symptomatique de cet intérêt renouvelé pour la période en général et pour le rôle tenu par l'Église espagnole en particulier, même si Helena Taberna se place résolument dans 
la défense d'une certaine église de culture basque en opposition avec la majorité des membres du clergé.

\section{Conclusion}

La récupération de la trame d'un film clérical franquiste, qui présentait l'Église comme la seule capable d'opérer la réconciliation de tous les Espagnols, permet de confronter les regards portés sur l'institution et d'approfondir la réflexion grâce aux liens intericoniques qui sous-tendent cette relecture. Mais Laurent Jenny relativise cette possibilité car le spectateur ne saura pas forcément repérer les liens intertextuels:

Le propre de l'intertextualité est d'introduire à un nouveau mode de lecture qui fait éclater la linéarité du texte. Chaque référence intertextuelle est le lieu d'une alternative: ou bien poursuivre la lecture en ne voyant là qu'un fragment comme un autre, qui fait partie intégrante de la syntagmatique du texte, ou bien retourner vers le texte d'origine (Jenny 1976: 227).

Il est certain que notre réflexion succincte sur les liens entre un film franquiste et La Buena Nueva doit s'étendre à d'autres champs. Pour prendre un dernier exemple de la richesse de ce film d'Helena Taberna, il se trouvera certainement des spectateurs pour percevoir la présence de photos d'Agustí Centelles derrière les scènes déchirantes de femmes qui pleurent leurs maris assassinés (Balsells 2001 : 137). On constate que la perception de l'intertextualité et de l'intericonicité dépend de la mémoire, de la culture et de la capacité analytique immédiate du spectateur. Dans bien des cas, il aura besoin de scruter l'œuvre plus d'une fois pour en déceler tous les secrets. La satisfaction intellectuelle qu'il en retirera en sera alors décuplée.

\section{Bibliographie citée}

Ayerra Redín, M., 1958, No me avergoncé del Evangelio (desde mi parroquia), Buenos Aires, Emecé.

Balsells, D. et al., 2001, La Guerre civile espagnole. Des photographes pour l'histoire, Barcelone, Museu Nacional d'Art de Catalunya.

Barthes, R., 1999, "Théorie du texte», in: Encyclopcedia Universalis, Paris, Encyclopædia Universalis.

Bernanos, G., 1936, Journal d'un curé de campagne, Paris, Plon.

Borges, J. L., 1997, «Tlön, Uqbar, Orbis Tertius», in: Ficciones, Madrid, Alianza.

Genette, G., 1982, Palimpsestes. La littérature au second degré, Paris, Seuil.

Giraudoux, J., 1949, Siegfried, I, 6, Paris, Grasset. 
Huete Machado, L., 2008, «El cura de Alsasua contra la Santa Cruzada», El País semanal, http://www.elpais.com/articulo/portada/cura/Alsasua/Santa/Cruzada/ elpepusoceps/20080102elpepspor_3/Tes. Dernière consultation le 12/01/2011. Jauss, H. R., 1978, Pour une esthétique de la réception, Paris, Gallimard.

Jenny, L., 1976, «La stratégie de la forme», Poétique, $27:$ 257-281.

Ricœur, P., 2004, «Le Juste, la justice et son échec» in Ricœur, Paris, Cahiers de l'Herne.

Riffaterre, M., 1981, «L'intertexte inconnu», Littérature, 41 : 4-5.

Sánchez-Vallejo, M.A., 2007, «Familiares de los curas vascos fusilados por Franco claman contra el olvido »,ElPaís, http://www.elpais.com/articulo/sociedad/somos/ nadie/elpepusoc/20071027elpepisoc_13/Tes. Dernière consultation le 12/01/2011. Seguin, J.-C., 1994, Histoire du cinéma espagnol, Paris, Nathan.

Sender, R. J., 1950, Réquiem por un campesino español, Barcelona, Destino.

Filmographie

Almodóvar, P., 2004, La mala educación.

Betriu, F., 1985, Réquiem por un campesino español.

Bresson, R., 1951, Journal d'un curé de campagne.

Cuerda, J. L., 2008, Los Girasoles ciegos.

Ford, J., 1941, How Green Was My Valley.

Gavras, C., 2002, Amen.

Gil, R., 1953, La guerra de Dios.

Nieves Conde, J.A., 1951, Balarrasa.

Nieves Conde, J.A., 1951, Surcos.

Poudovkine, V., 1926, Mamb.

Taberna, H, 2007, La Buena Nueva.

Vidor, K., 1938, The Citadel. 